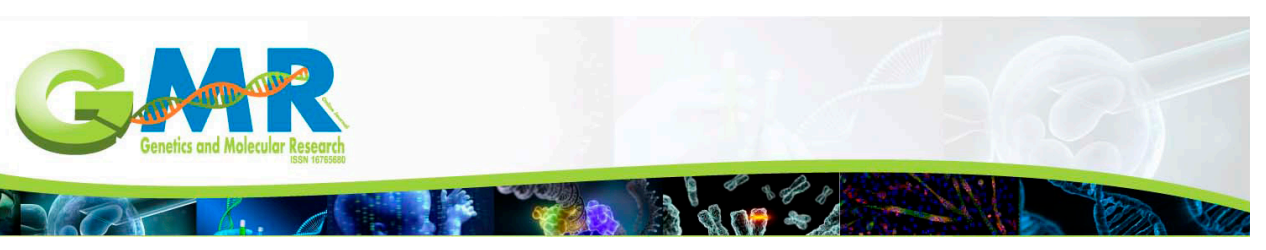

\title{
Frequency of gray coat color in native Chinese horse breeds
}

\author{
K.X. Gao', N.B. Chen', W.J. Liu², R. Li', X.Y. Lan', H. Chen', \\ C.Z. Lei ${ }^{1}$ and R.H. Dang ${ }^{1}$ \\ ${ }^{1}$ College of Animal Science and Technology, Northwest A\&F University, \\ Yangling, Shaanxi, China \\ ${ }^{2}$ College of Animal Science, Xinjiang Agricultural University, \\ Urumqi, Xinjiang, China \\ Corresponding authors: R.H. Dang / W.J. Liu \\ E-mail: dangruihua@nwsuaf.edu.cn / Iwj-ws@163.com
}

Genet. Mol. Res. 14 (4): 14144-14150 (2015)

Received June 26, 2015

Accepted August 25, 2015

Published October 29, 2015

DOI http://dx.doi.org/10.4238/2015.October.29.36

ABSTRACT. Gray horses are born colored, and they then gradually lose their hair pigmentation. Tremendous progress has been made in identifying the genes responsible for graying with age in horses in recent years. Results show that gray coat color in horses is caused by a 4.6-kb duplication in intron 6 of the syntaxin 17 gene (STX17), which constitutes a cis-acting-regulatory mutation. However, little is known about the gray phenotype in native Chinese horses. This study was conducted to explore the frequency distribution of the gray mutation in native Chinese horse breeds. A total of 489 samples from 14 native Chinese horse breeds were genotyped for the STX17 duplication using a simplified conventional PCR-based method. The results show that the gray mutation was present in 12 native Chinese horse breeds, except the Balikun and Guanzhong breeds. The Chakouyi and Hequ breeds had the highest frequency of the gray mutation $\left(P_{G}=0.367\right.$ and $P_{G}=0.274$, respectively). There was no significant geographical difference in the distribution of gray coat color 
across native Chinese horse breeds. Our results suggest that gray is a common coat color in Chinese horses.

Key words: Chinese horse; Simplified PCR-based method; Graying with age

\section{INTRODUCTION}

As one of the earliest domesticated animals, the horse has had a large impact on warfare, transportation, and communication capability (Ludwig et al., 2009). Horses have various coat colors, including white, black, brown, and bay, which have been strongly selected by humans throughout history (Fang et al., 2009). Compared to horses with other coat colors, white-coated horses have had an important impact on art and literature across the world for centuries and are considered to be powerful and holy, and to possess dignity (Tresidder, 2005). Therefore, humans have selected the "white" phenotype in horse breeding over hundreds of generations.

Gray horses are born colored and they then progressively turn gray, eventually reaching a uniform white coat color by 6 to 8 years of age, and their skin remains dark (Curik et al., 2013). Graying with age is one of the most common causes of white coat color and is widely distributed among many horse breeds, which has led to the selection of gray coat color by humans for many years (Sponenberg, 2009). Graying with age is an autosomal dominant trait. Previous studies have shown that graying with age is caused by a 4.6-kb intronic duplication in the syntaxin 17 gene (STX17) in horses (Pielberg et al., 2008). This cis-acting-regulatory mutation is also associated with a high incidence of melanoma and vitiligo-like depigmentation in gray horses (Pielberg et al., 2008). The gray mutation can strongly influence melanoma, vitiligo, and speckling grade, and capacitate the effects of the loss-of-function mutation at the agouti (ASIP) locus on melanoma grade (Curik et al., 2013). Melanoma occurs frequently in gray horses, usually around the eyes, genital regions, or the anus (Fleury et al., 2000), and these tumors are often malignant (Laus et al., 2010). Almost $80 \%$ of gray horses develop melanomas if they live beyond 15 years of age (Seltenhammer et al., 2004). All these aspects make the gray horse a good model in which to study the inheritance of traits with a complex genetic background (Curik et al., 2013). Results show that the 4.6-kb intronic duplication is the only polymorphism uniquely associated with the gray phenotype, as it was exclusively detected in all gray horses but not in any of non-gray horses (Sundstrom et al., 2012).

China has more than 29 native horse breeds with various coat colors. However, there are no reports of mutations related to graying with age in Chinese horses. The aims of this study were to i) determine whether the duplication in STX17 is associated with the gray phenotype of 19 Chakouyi horses, ii) genotype 489 individuals (including 19 Chakouyi horses) across 14 native Chinese horse breeds using PCR, and iii) investigate the geographical distribution of the gray locus covering north-western, central, and north China.

\section{MATERIAL AND METHODS}

\section{Sample collection and DNA extraction}

In total, 470 blood samples (278 males, 192 females) were randomly collected from 14 
Chinese horse breeds (Table 1). Sample collection was approved by the owners. Genomic DNA was extracted using the standard phenol-chloroform method (Sambrook et al., 1989). The coat color record of 19 Chakouyi horses (ten gray and nine non-gray horses) was obtained for validating the diagnostic genotyping tests.

\begin{tabular}{|c|c|c|c|c|c|c|c|c|}
\hline \multirow[t]{2}{*}{ Type } & \multirow[t]{2}{*}{ Breed } & \multirow[t]{2}{*}{$\begin{array}{l}\text { Sample } \\
\text { size }\end{array}$} & \multirow[t]{2}{*}{$\begin{array}{l}\text { Number of } \\
\text { gray horses }\end{array}$} & \multicolumn{3}{|c|}{$\begin{array}{l}\text { Genotype frequency } \\
\text { (number) }\end{array}$} & \multicolumn{2}{|c|}{$\begin{array}{c}\text { Allele } \\
\text { frequency }\end{array}$} \\
\hline & & & & $P(G / G)$ & $P(G / g)$ & $P(g / g)$ & $P(G)$ & $P(g)$ \\
\hline \multirow[t]{5}{*}{ Kazakh } & Kazakh (KZK) & 17 & 1 & $0.000(0)$ & $0.059(1)$ & $0.941(16)$ & 0.029 & 0.971 \\
\hline & Balikun (BLK) & 17 & 0 & $0.000(0)$ & $0.000(0)$ & $1.000(17)$ & 0.000 & 1.000 \\
\hline & Debao bony (DB) & 40 & 13 & $0.100(4)$ & $0.225(9)$ & $0.675(27)$ & 0.213 & 0.788 \\
\hline & Guizhou (GZ) & 40 & 5 & $0.050(2)$ & $0.075(3)$ & $0.875(35)$ & 0.088 & 0.913 \\
\hline & Lichuan (LC) & 22 & 1 & $0.000(0)$ & $0.045(1)$ & $0.955(21)$ & 0.023 & 0.977 \\
\hline \multirow[t]{5}{*}{ Southwest } & Guanzhong (GU) & 24 & 0 & $0.000(0)$ & $0.000(0)$ & $1.000(24)$ & 0.000 & 1.000 \\
\hline & Ningqiang (NQ) & 41 & 3 & $0.000(0)$ & $0.073(3)$ & $0.927(38)$ & 0.037 & 0.963 \\
\hline & Baise (BS) & 35 & 7 & $0.057(2)$ & $0.143(5)$ & $0.800(28)$ & 0.129 & 0.871 \\
\hline & Chaidamu (CD) & 46 & 8 & $0.000(0)$ & $0.174(8)$ & $0.826(38)$ & 0.087 & 0.913 \\
\hline & Datong (DT) & 28 & 9 & $0.036(1)$ & $0.286(8)$ & 0.679 (19) & 0.179 & 0.821 \\
\hline \multirow[t]{2}{*}{ Mongolia } & Yanji (YJ) & 43 & 8 & $0.000(0)$ & $0.186(8)$ & $0.814(35)$ & 0.093 & 0.907 \\
\hline & Chakouyi (CKY) & 49 & 24 & $0.245(12)$ & $0.245(12)$ & $0.510(25)$ & 0.367 & 0.633 \\
\hline Hequ & Hequ (HQ) & 31 & 9 & $0.258(8)$ & $0.032(1)$ & $0.710(22)$ & 0.274 & 0.726 \\
\hline Tibet & Yushu (YS) & 56 & 10 & $0.179(10)$ & $0.000(0)$ & $0.821(46)$ & 0.179 & 0.821 \\
\hline Total & & 470 & 98 & $0.080(39)$ & $0.121(59)$ & $0.800(391)$ & 0.140 & 0.860 \\
\hline
\end{tabular}

\section{PCR amplification and genotyping}

Long-range PCR using the primer 2 pair (DupForward and DupReverseD) (Pielberg et al., 2008) was used to identify the gray horses (Table 2). The 4867-bp PCR product generated in gray horses was relatively long, and could lead to the failure of PCR amplification because of the need for high-quality DNA template and special Taq polymerase. Thus, based on sequence data (EU606026 and EU606027), the special primer 1 pair was designed to amplify the genomic region containing allele $G$, which allows quick discrimination between gray ( $G / G, G / g)$ and non-gray $(\mathrm{g} / \mathrm{g})$ horses. Nineteen Chakouyi horses were analyzed using PCR with primer 1 and the published primer 2 (Table 2).

Table 2. Primers for PCR analysis of the syntaxin 17 gene (STX17) in gray and non-gray horses

\begin{tabular}{lllc}
\hline & & \multicolumn{1}{c}{ Sequence } & Reference \\
\hline Primer 1 & Grey-F & 5'-GCTCATTAGAAATGCAAAATCTCAG-3' & This study \\
& Grey-R & 5'-AGTAATCAGAGGTTCTCCTTTGAGC-3' & Pielberg et al. (2008) \\
& DupForward & 5'-GGAACATAAAGTAGATTTGGTGGGAAAG-3' & \\
& DupReverseN & 5'-TTCTGATAAATGCATAAACCCACGTAAC-3' & \\
& DupReverseD & 5'-TTCCAATTCTGAGATTTTGCATTTCTAA-3' & \\
\hline
\end{tabular}

Normal PCR amplification with primer 1 was performed in a $25-\mu \mathrm{L}$ reaction, containing $12.5 \mu \mathrm{L}$ 2X Taq MasterMix (TaKaRa, Dalian, China) (including Taq DNA polymerase, 2X PCR buffer, $3 \mathrm{mM} \mathrm{MgCl}_{2}$, and $400 \mu \mathrm{M}$ dNTP mix), $0.2 \mu \mathrm{M}$ of each primer, and $40 \mathrm{ng}$ template DNA. The PCR conditions were $5 \mathrm{~min}$ at $95^{\circ} \mathrm{C}$, followed by 33 cycles for $40 \mathrm{~s}$ at $94^{\circ} \mathrm{C}, 40 \mathrm{~s}$ at $63^{\circ} \mathrm{C}$ annealing temperature, $70 \mathrm{~s}$ at $72^{\circ} \mathrm{C}$, and a final extension of $10 \mathrm{~min}$ at $72^{\circ} \mathrm{C}$. PCR products were electrophoresed on $1.5 \%$ agarose gels. 
Long-range PCR with primer 2 was performed in a $20-\mu \mathrm{L}$ reaction containing $40 \mathrm{ng}$ template DNA, 0.5 U PrimeSTAR GXL DNA polymerase (TaKaRa), $0.2 \mu \mathrm{M}$ of each primer, $1.6 \mu \mathrm{L}$ dNTP mixture (2.5 mM each), and $4 \mu \mathrm{L} 5 \mathrm{X}$ PrimeSTAR GXL buffer. Long-range PCR included 33 cycles for $10 \mathrm{~s}$ at $98^{\circ} \mathrm{C}, 15 \mathrm{~s}$ at $60^{\circ} \mathrm{C}, 6 \mathrm{~min}$ at $68^{\circ} \mathrm{C}$, and then $10 \mathrm{~min}$ at $68^{\circ} \mathrm{C}$ as a final extension. The products were electrophoresed on $1 \%$ agarose gels. The long-range PCR can be used to identify the genotypes $(\mathrm{G} / \mathrm{G}, \mathrm{G} / \mathrm{g})$ of gray horses.

\section{RESULTS}

\section{Identification of gray-specific primer 1 in 19 Chakouyi horses}

Normal PCR with primer 1 and long-range PCR with primer 2 (DupForward and DupReverseD) were used to genotype the 19 Chakouyi horses (ten gray and nine non-gray horses) first. The results using the two different primer pairs were consistent, with either 5415- or 1085-bp products only generated in gray horses (Figure 1), except one sample (data not shown). The specificity of the short and the long PCR products was also confirmed by sequencing. Based on this, primer 1 was able to detect the gray mutation effectively.
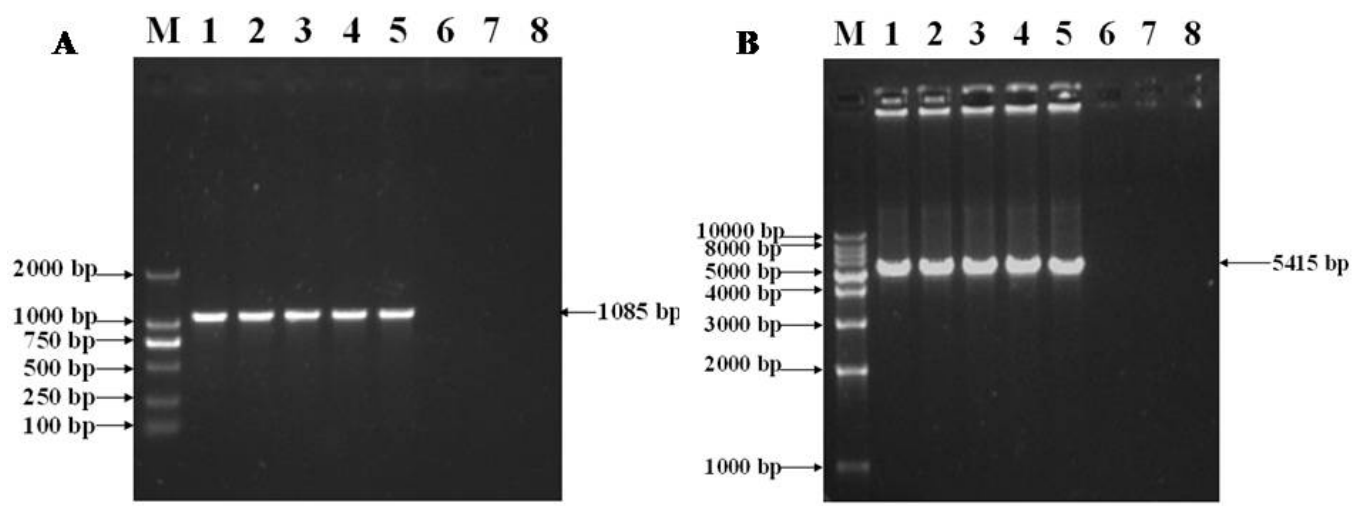

Figure 1. Genotyping of the syntaxin 17 gene (STX17) mutation in gray Chinese horses using PCR. A. Genotyping gray and non-grey horses using Primer 1. Lane $M=$ marker; lanes 1-5 = individuals with gray coat color; lanes 6 and 7 = individuals with non-gray coat color; lane $8=$ blank. Only gray horses have the 1085-bp PCR product. B. Genotyping heterozygote $(\mathrm{G} / \mathrm{g})$ and homozygous $(\mathrm{G} / \mathrm{G})$ individuals in gray horses. Lane $M=$ marker; lanes $1-5=$ individuals with genotype $\mathrm{G} / \mathrm{g}$; lanes 6 and 7 = individuals with genotype $\mathrm{G} / \mathrm{G}$; lane 8 = blank. In Chinese gray horses, only heterozygotes $(\mathrm{G} / \mathrm{g})$ have the 5415 -bp PCR product.

\section{Genotypic and allelic frequencies}

In this analysis, primer 2 (DupForward and DupReverseN) was used to identify the normal sequence $(\mathrm{g})$ and primer 1 was used to identify the duplicate sequence $(G)$. In total, 98 of the $489(20.04 \%)$ Chinese horses sampled had gray coat color. Of these 98 gray horses, 39 were homozygote $(\mathrm{G} / \mathrm{G})$ and 59 were heterozygote $(\mathrm{G} / \mathrm{g}$ ) (Table 1). The genotype frequencies of $\mathrm{G} / \mathrm{G}$, $\mathrm{G} / \mathrm{g}$, and $\mathrm{g} / \mathrm{g}$ were $0.080,0.121$, and 0.800 , respectively. The allele frequencies of $\mathrm{G}$ and $\mathrm{g}$ were 0.140 and 0.860 , respectively. Gray coat color was found in 12 of the 14 breeds assessed. The Chakouyi horse had the highest frequency of allele $\mathrm{G}\left(P_{G}=0.367\right)$ and the Hequ horse had the 
highest frequency of $\mathrm{G} / \mathrm{G}$ among the 12 native Chinese breeds $\left(P_{G / G}=0.258\right)$. In addition, all Kazakh, Lichuan, Ningqiang, Chaidamu, and Yanji horses with gray coat color were heterozygous with the G/g genotype, whereas all Yushu horses with gray coat color were homozygous (G/G).

\section{Geographical distribution of gray coat horses}

The geographical distribution of the phenotype frequency of the gray mutation showed that the gray locus was widely distributed across native Chinese horse breeds, except the Balikun and Guanzhong breeds (Figure 2). There was no significant geographical difference in the distribution of gray coat color across 12 native Chinese horse breeds.

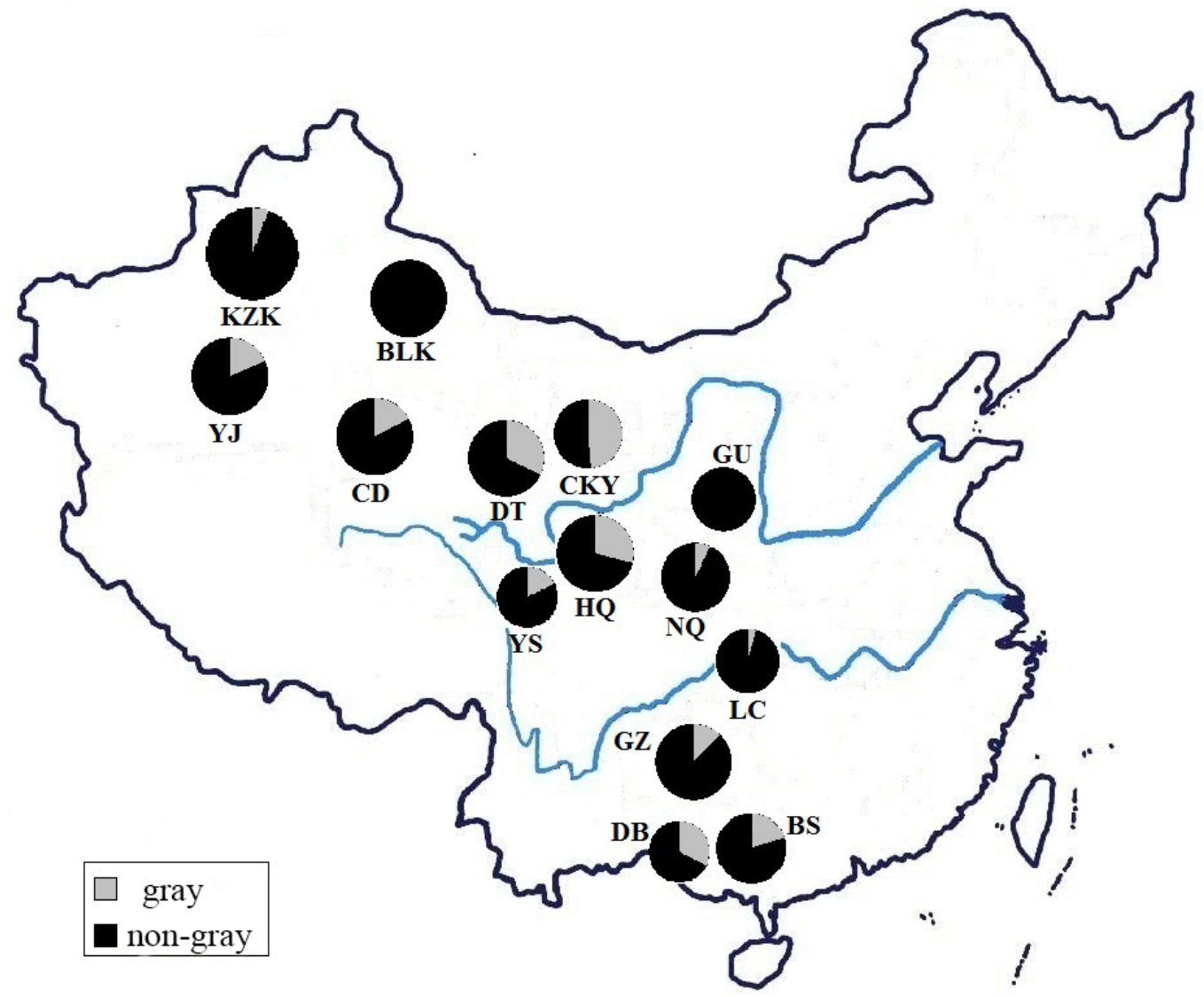

Figure 2. Geographical distribution of the gray phenotype among Chinese horse breeds.

\section{DISCUSSION}

In the present study, a new primer pair was designed to effectively detect the gray coat color mutation in horse. The new primer pair was verified on samples from ten gray and nine non-gray Chakouyi horses. The amplification products from the two primer pairs were consistent 
with the phenotypes in 19 Chakouyi individuals, except one. The gray mutation is an autosomal dominant trait and horses with gray coat color are born colored, then gradually become gray and, by the age of 6 to 8 years, develop a white coat color (Curik et al., 2013). As a consequence, it is difficult for us to obtain the accurate phenotype of a young gray horse, even if the parents' coat color is known. Thus, the identification of one Chakouyi gray horse may be incorrect, but the others were correct. The results demonstrate that the 4.6-kb duplication in intron 6 of STX17 is the cause of gray coat color in native Chinese horses, which is consistent with horses in other countries (Pielberg et al., 2008).

In the wild, light-coated animals have a poor survival rate during evolution because they are easily detected by predators. However, the percentage of native Chinese horses with gray coat color in the current study was relatively high (20.04\%). A previous study showed that light coat color horses were less attractive to blood-sucking tabanid flies compared with dark horses (Horvath et al., 2010). In addition, all gray horses have the advantage of their dark skin pigmentation against UV radiation (Sundstrom et al., 2012), which helps gray horses to survive in the wild. Furthermore, as one of the most common causes of white color in horses (Sponenberg, 2009), the gray phenotype has also been artificially selected by humans, which has increased the frequency of the mutated $G$ allele. These aspects improve the survival of gray horses in the wild. In the current study, 39 horses with a homozygote genotype were detected in 98 of the gray horses, thus, $39.80 \%$ of the Chinese gray horses were homozygotes. Pielberg et al. (2008) reported that the progression of coat color to completely white at maturity is more rapid in homozygous horses with the gray mutation than in heterozygous gray horses. This may have led to selection of homozygous gray horses by humans.

Native Chinese horses have various coat colors, including bay, black, white, and gray. Gray horses appear in many breeds, although the color is most commonly seen in breeds descended from Arabian ancestors. In the current study, the gray mutation was detected in 12 of the 14 breeds assessed, and had no significant geographical distribution. In addition, the Chakouyi horse had the highest frequency of allele $\mathrm{G}\left(P_{G}=0.367\right)$ among the 12 breeds. This observation suggests that the Chakouyi horse has a large number of gray-colored horses. However, the gray mutation was not detected in Balikun and Guanzhong horses, which indicates that these two horse breeds may not have the gray coat color or the gray coat color is very rare in these two breeds. The observations from the current study suggest that gray is one of the most common coat colors in Chinese horses. A previous study hypothesized that gray horses in Europe have a common ancestor with the mutated G allele (Pielberg et al., 2008). Our results suggest that horses with gray coat color in China may date from a common ancestor with a gray mutation in the STX17 gene. Therefore, we suppose that graying with age is an ancient trait occurring around the world.

\section{CONCLUSION}

In this study, we found that the 4.6-kb duplication in intron 6 of STX17 was associated with the gray phenotype of native Chinese horses, which implies that Chinese and gray horses in other countries had a common ancestor during evolution. Gray coat color was found to exist widely in native Chinese horses. Our research may provide a genetic basis for the selection of coat color in horses.

\section{Conflicts of interest}

The authors declare no conflict of interest. 


\section{ACKNOWLEDGMENTS}

Research supported by the National Natural Science Foundation of China (\#31072001, \#81270439), the National Key Technology Support Program (\#2012BAD44B01), and the Xinjiang Major Scientific and Technological Program (\#201130101-3).

\section{REFERENCES}

Curik I, Druml T, Seltenhammer M, Sundstrom E, et al. (2013). Complex inheritance of melanoma and pigmentation of coat and skin in Gray horses. PLoS Genet. 9: e1003248.

Fang M, Larson G, Ribeiro HS, Li N, et al. (2009). Contrasting mode of evolution at a coat color locus in wild and domestic pigs. PLoS Genet. 5: e1000341.

Fleury C, Berard F, Balme B and Thomas L (2000). The study of cutaneous melanomas in Camargue-type gray-skinned horses (1): clinical-pathological characterization. Pigment Cell Res. 13: 39-46.

Horvath G, Blaho M, Kriska G, Hegedus R, et al. (2010). An unexpected advantage of whiteness in horses: the most horseflyproof horse has a depolarizing white coat. Proc. Biol. Sci. 277: 1643-1650.

Laus F, Cerquetella M, Paggi E, Ippedico G, et al. (2010). Evaluation of cimetwine as a therapy for dermal melanomatosis in gray horse. Isr. J. Vet. Med. 65: 48-52.

Ludwig A, Pruvost M, Reissmann M, Benecke N, et al. (2009). Coat color variation at the beginning of horse domestication. Science 324: 485.

Pielberg GR, Golovko A, Sundstrom E, Curik I, et al. (2008). A cis-acting regulatory mutation causes premature hair graying and susceptibility to melanoma in the horse. Nat. Genet. 40: 1004-1009.

Sambrook J, Fritsch EF and Maniatis T (1989). Molecular Cloning: a laboratory manual. 2nd edn. Cold Spring Harbor Laboratory Press, Cold Spring Harbor.

Seltenhammer MH, Heere-Ress E, Brandt S, Druml T, et al. (2004). Comparative histopathology of gray-horse-melanoma and human malignant melanoma. Pigment Cell Res. 17: 674-681.

Sponenberg DP (2009). Equine color genetics. Wiley-Blackwell, lowa.

Sundstrom E, Komisarczuk AZ, Jiang L, Golovko A, et al. (2012). Identification of a melanocyte-specific, microphthalmiaassociated transcription factor-dependent regulatory element in the intronic duplication causing hair graying and melanoma in horses. Pigment Cell Melanoma Res. 25: 28-36.

Tresidder J (2005). The complete dictionary of symbols. Chronicle Books. Raincoast Books, Vancouver. 